\title{
Parameterized thermal macromodeling for fast and effective design of electronic components and systems
}

\author{
F. Ferranti, T. Dhaene \\ Dept. of Information Technology (INTEC) \\ Ghent University-iMinds \\ Gaston Crommenlaan 8 Bus 201, \\ 9050 Ghent, Belgium \\ Email: francesco.ferranti@intec.ugent.be
}

\author{
S. Russo \\ Meridiana Italia S.r.l. \\ via Sicilia 50, 00187 Rome, Italy
}

\author{
A. Magnani, M. de Magistris, \\ V. d'Alessandro, and N. Rinaldi \\ Dept. Electrical Engineering and \\ Information Technology \\ University Federico II, \\ via Claudio 21, 80125 Naples, Italy
}

\begin{abstract}
We present a parameterized macromodeling approach to perform fast and effective dynamic thermal simulations of electronic components and systems where key design parameters vary. A decomposition of the frequency-domain data samples of the thermal impedance matrix is proposed to improve the accuracy of the model and reduce the number of the computationally costly thermal simulations needed to build the macromodel. The methodology is successfully applied to analyze the impact of layout variations on the dynamic thermal behavior of a state-of-the-art 8-finger AlGaN/GaN HEMT grown on a SiC substrate.
\end{abstract}

\section{INTRODUCTION}

Thermal effects are becoming a major issue in modern electronic components and systems due to multiple reasons, e.g., increase in power density induced by high integration levels, use of insulation schemes based on silicon dioxide, and adoption of advanced materials suffering from poor thermal conductivity (e.g., GaAs and $\mathrm{AlGaN}$ ) [1], [2]. As a result, both reliability and performance might be jeopardized if countermeasures are not taken. The thermal design can be improved by resorting to accurate electrothermal simulations; on the other hand, the choice of the approach to account for the thermal feedback (TF) is still challenging. A well-known strategy relies on the identification of reduced equivalent electrical networks - compatible with commercial circuit simulators - to describe the heat propagation through the component/system at points of interest. However, the identification of the components embedded in such networks requires a number of CPU/memory demanding 3-D thermal simulations (in principle one for each heat source) using numerical thermal solvers based on e.g., the finite element method (FEM), in order to evaluate the thermal impedance matrix [3]. Moreover, if crucial design activities are to be carried out (e.g., design space exploration, optimization, and variability analysis), both the mesh generation (if FEM is adopted) and the whole set of simulations must be repeated for several values of design parameters (e.g., layout features), which implies a huge computational cost.

Parameterized (also called parametric or scalable) macromodels can be used to speed-up design steps without compromising the reliability and accuracy of the results. These models are well-suited to describe the system behavior at the input/output ports (e.g., scattering, admittance and impedance input/output representations) as a function of frequency and design parameters. Their construction requires a limited amount of computationally expensive numerical simulations. Over the years, different parameterized macromodeling techniques have been proposed [4]-[9], which have been applied to a broad range of systems, such as high-speed interconnects, microwave filters, spiral inductors.

In this paper, for the first time a parameterized macromodeling approach is used for efficient thermal simulations of electronic components and systems. The macromodels were generated by means of the method presented in [8]. Moreover, a decomposition of numerical data samples of the thermal impedance matrix in the frequency domain is proposed, which allows improving the modeling accuracy and reducing the number of onerous numerical simulations needed for the macromodel generation. The proposed methodology is successfully validated by examining the influence of layout variations on the dynamic thermal behavior of a state-of-the-art multi-finger AlGaN/GaN High-Electron Mobility Transistors (HEMTs) grown on a $\mathrm{SiC}$ substrate.

\section{PARAMETERIZED MACROMODELING TECHNIQUE}

The technique [8] generates a parameterized macromodel $\mathbf{H}_{\text {model }}(s, \mathbf{g})$ to accurately represent a set of multidimensional data samples $\left\{\left(s_{f}, \mathbf{g}_{k}\right), \mathbf{H}\left(s_{f}, \mathbf{g}_{k}\right)\right\}, f=1, \ldots, F, k=$ $1, \ldots, K_{t o t}$ which depend on the complex frequency $s=j \omega$ and $M$ design variables $\mathbf{g}=\left(\mathrm{g}^{(m)}\right)_{m=1}^{M}$, such as layout or substrate features. A parameterized macromodel in a pole-residue form

$$
\mathbf{H}_{\text {model }}(s, \mathbf{g})=\mathbf{C}_{0}(\mathbf{g})+\sum_{n=1}^{N(\mathbf{g})} \frac{\mathbf{C}_{n}(\mathbf{g})}{s-p_{n}(\mathbf{g})}
$$

or in a state-space form

$$
\mathbf{H}_{\text {model }}(s, \mathbf{g})=\mathbf{C}(\mathbf{g})(s \mathbf{I}-\mathbf{A}(\mathbf{g}))^{-1} \mathbf{B}(\mathbf{g})+\mathbf{D}(\mathbf{g})
$$

is computed. The design space contains all design parameters g and two data design space grids are used in the modeling process: an estimation grid and a validation grid. The estimation grid is adopted to build a parameterized macromodel, while the validation grid is employed to verify its modeling capability in a set of points of the design space previously not used for the model generation. The method in [8] first identifies a set of rational univariate macromodels $\mathbf{H}_{\text {model }}\left(s, \mathbf{g}_{k}\right)$, which are called root macromodels, at the estimation design space points. Then, the estimation design space grid is divided into cells using hyperrectangles (regular grids) or simplices (regular and scattered grids).

The validation set points are located at the center of the cells of the estimation grid. Fig. 1 shows a possible 2-D 
estimation and validation set with rectangular grid cells.

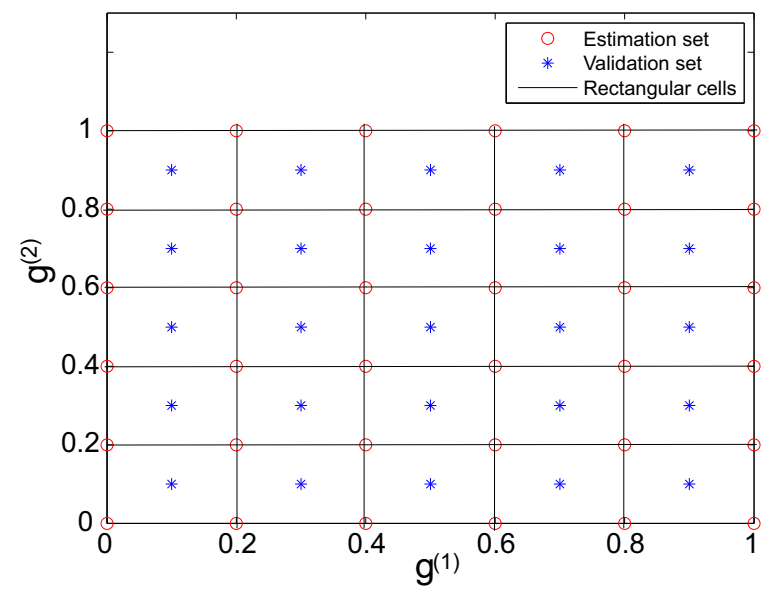

Fig. 1. Estimation and validation set with rectangular grid cells (normalized $\mathrm{g}^{(1)}, \mathrm{g}^{(2)}$ are considered).

Once the estimation design space grid is divided into cells, a local parameterized model is associated to each cell that is a subdomain of the entire design space. We indicate a cell region of the design space as $\Omega_{i}, i=1, \ldots, P$ and the corresponding vertices as $\mathbf{g}_{k}^{\Omega_{i}}, k=1, \ldots, Q$. We note that each vertex corresponds to a root macromodel $\mathbf{H}_{\text {model }}\left(s, \mathbf{g}_{k}^{\Omega_{i}}\right)$. For each cell, an optimization procedure is used to find amplitude and frequency scaling coefficients that make each vertex an accurate approximant of the other cell vertices (an error function to be minimized regulates the quality of this approximation). For each vertex $\mathbf{H}_{\text {model }}\left(s, \mathbf{g}_{k}^{\Omega_{i}}\right)$, a set of amplitude $\alpha_{1, k}\left(\mathbf{g}_{j}^{\Omega_{i}}\right), j=$ $1, \ldots, Q$ and frequency $\alpha_{2, k}\left(\mathbf{g}_{j}^{\Omega_{i}}\right), j=1, \ldots, Q$ scaling real coefficients are calculated

$$
\min _{\alpha_{1, k}\left(\mathbf{g}_{j}^{\Omega_{i}}\right), \alpha_{2, k}\left(\mathbf{g}_{j}^{\Omega_{i}}\right)} \operatorname{Err}\left(\widetilde{\mathbf{H}}_{\text {model }}\left(s, \mathbf{g}_{k}^{\Omega_{i}}\right), \mathbf{H}_{\text {model }}\left(s, \mathbf{g}_{j}^{\Omega_{i}}\right)\right)
$$

with

$$
\begin{gathered}
\widetilde{\mathbf{H}}_{\text {model }}\left(s, \mathbf{g}_{k}^{\Omega_{i}}\right)=\alpha_{1, k}\left(\mathbf{g}_{j}^{\Omega_{i}}\right) \mathbf{H}_{\text {model }}\left(s \alpha_{2, k}\left(\mathbf{g}_{j}^{\Omega_{i}}\right), \mathbf{g}_{k}^{\Omega_{i}}\right) \\
\alpha_{1, k}\left(\mathbf{g}_{j}^{\Omega_{i}}\right)=\alpha_{2, k}\left(\mathbf{g}_{j}^{\Omega_{i}}\right)=1, j=k \\
\alpha_{1, k}\left(\mathbf{g}_{j}^{\Omega_{i}}\right) \geq 0 \\
\alpha_{2, k}\left(\mathbf{g}_{j}^{\Omega_{i}}\right)>0
\end{gathered}
$$

Finally, positive interpolation schemes are used to parameterize the root macromodels and corresponding scaling coefficients and then to generate a parameterized model $\mathbf{H}_{\text {model }}(s, \mathbf{g})$. Further details about this macromodeling technique can be found in [8] and are not repeated here for the lack of space.

In this paper, parameterized macromodels for the thermal behavior of electronic components and systems are investigated. The thermal impedance matrix [3] is modeled as a function of frequency and additional design parameters $\mathbf{g}$. A decomposition of the frequency-domain data samples of the thermal impedance matrix is presented to enhance the modeling accuracy and limit the computational cost of the simulations needed for the macromodel generation. Considering the set of thermal impedance matrices at the estimation points $\mathbf{Z}\left(s_{f}, \mathbf{g}_{k}\right)$, the corresponding DC value $\mathbf{R}\left(\mathbf{g}_{k}\right)$ is extracted and the initial impedance data samples are pre-processed as

$$
\widehat{\mathbf{Z}}\left(s_{f}, \mathbf{g}_{k}\right)=\mathbf{Z}\left(s_{f}, \mathbf{g}_{k}\right) \circ \mathbf{G}\left(\mathbf{g}_{k}\right)
$$

where $\circ$ denotes the Hadamard product [10] (entrywise product of two matrices of the same size) and $\mathbf{G}\left(\mathbf{g}_{k}\right)$ is the Hadamard inverse of $\mathbf{R}\left(\mathbf{g}_{k}\right)$ and therefore each of its entries is

$$
G_{i j}\left(\mathbf{g}_{k}\right)=\left(R_{i j}\left(\mathbf{g}_{k}\right)\right)^{-1}
$$

The matrix $\mathbf{R}$ is real, symmetric with all positive elements and positive definite. Two parameterized macromodels are generated, $\widehat{\mathbf{Z}}_{\text {model }}(s, \mathbf{g})$ and $\mathbf{R}_{\text {model }}(\mathbf{g})$ starting from the data samples $\widehat{\mathbf{Z}}\left(s_{f}, \mathbf{g}_{k}\right)$ and $\mathbf{R}\left(\mathbf{g}_{k}\right)$, respectively. $\mathbf{R}_{\text {model }}(\mathbf{g})$ does not depend on frequency and therefore a parameterized macromodel can be built using standard interpolation/approximation models (e.g., radial basis functions, polynomials, splines, etc.). Once both parameterized macromodels are generated, the model $\mathbf{Z}_{\text {model }}(s, \mathbf{g})$ representing the original thermal impedance data samples can be expressed as

$$
\mathbf{Z}_{\text {model }}(s, \mathbf{g})=\widehat{\mathbf{Z}}_{\text {model }}(s, \mathbf{g}) \circ \mathbf{R}_{\text {model }}(\mathbf{g})
$$

Considering a pole-residue form for $\widehat{\mathbf{Z}}_{\text {model }}(s, \mathbf{g})$

$$
\widehat{\mathbf{Z}}_{\text {model }}(s, \mathbf{g})=\mathbf{C}_{0}(\mathbf{g})+\sum_{n=1}^{N(\mathbf{g})} \frac{\mathbf{C}_{n}(\mathbf{g})}{s-p_{n}(\mathbf{g})}
$$

then (10) can be written as

$$
\begin{aligned}
& \mathbf{Z}_{\text {model }}(s, \mathbf{g})= \\
& =\mathbf{C}_{0}(\mathbf{g}) \circ \mathbf{R}_{\text {model }}(\mathbf{g})+\sum_{n=1}^{N(\mathbf{g})} \frac{\mathbf{C}_{n}(\mathbf{g}) \circ \mathbf{R}_{\text {model }}(\mathbf{g})}{s-p_{n}(\mathbf{g})}
\end{aligned}
$$

It is worth remarking that this data decomposition is important for two main reasons:

- it allows enhancing the modeling accuracy of the macromodel;

- $\quad$ it allows extracting the DC information of the thermal impedance matrix and modeling it separately. The computational resources needed to evaluate the data samples of the frequency-dependent thermal response $\mathbf{Z}\left(s_{f}, \mathbf{g}_{k}\right)$ (dynamic thermal simulations) are much more significant than in the case of the computation of only the related DC value $\mathbf{R}\left(\mathbf{g}_{k}\right)$ (steady-state thermal simulations). The sampling in the design space to get dynamic and steady-state thermal response data samples can be decoupled, which helps reduce the overall computational cost to build a parameterized macromodel.

\section{CASE STUDY}

HEMTs are unipolar field-effect devices where the current conduction is due to a 2-D electron gas flowing through a low-resistivity thin undoped layer (also referred to as channel) located at the junction between two materials with different bandgaps. In this layer, high mobility is reached since the carriers are not subject to collisions with doping impurities (ionized-impurity scattering), which instead occur in conventional transistors. In addition, HEMTs enjoy outstanding properties like high breakdown field and high saturation drift velocity. All these benefits make such devices attractive for high-frequency applications where high gain and low noise are required, like radars operating in extreme environments, microwave communications, and radio astronomy [11]. In particular, extensive research effort has been recently focused on multi-finger (i.e., multi-gate) AlGaN/GaN HEMTs, 
which offer the highest output power and are considered as the most appealing devices for microwave power amplifiers [12]. However, these transistors suffer from high channel temperatures induced by the fast designer-induced growth in current (and power) density due to higher bandwidth signals requirements for modern communications. Thermal effects can be minimized by a proper layout optimization, which can in turn be accomplished by making use of the proposed time-saving macromodeling approach. An $\mathrm{M}_{\mathrm{hs}}$-finger HEMT exhibits $\mathbf{M}_{\mathrm{hs}}$ regions in which the power is dissipated (i.e., $\mathbf{M}_{\mathrm{hs}}$ heat sources); as a consequence, this device can be associated to an $\mathrm{M}_{\mathrm{hs}} \times \mathrm{M}_{\mathrm{hs}}$ matrix of thermal impedances $\mathrm{Z}_{\mathrm{ij}}(\mathrm{t})$ where $\mathrm{t}$ denotes the time variable $\left(Z_{\mathrm{ii}}\right.$ are the self-heating impedances, whereas $Z_{i j(i \neq j)}$ are the mutual ones). Such a matrix should be in principle determined by means of $\mathbf{M}_{\mathrm{hs}}$ accurate, yet resource demanding, 3-D numerical simulations [13]. The numerical analysis was supported by an in-house code that allows automatically drawing the 3-D transistor structure and building the mesh of the device within the environment of the commercial FEM software package Comsol [14]. The resulting mesh was further optimized by invoking smart refinement techniques available in the latest software releases, eventually leading to about $2.5 \times 10^{5}$ elements (tetrahedra). An example of the mesh used by Comsol is shown in Fig. 2. The evaluation of the dynamic temperature field within the whole structure due to the activation of a single heat source requires about 3 hours on a workstation equipped with 2 hexa-core Intel Xeon E7450 CPUs and 100 GB RAM. Exploiting the structure symmetry, only 4 transient simulations are needed to compute the thermal impedance matrix, requiring a total of about 12 hours (for a fixed layout configuration). It is worth noting that the thermal impedance is obtained through transient simulations in logarithmically-spaced samples; however, in order to use the macromodeling strategy, the FEM data have to be preliminarily converted in the frequency domain, as in [3]. The key layout parameters are the gate width $\mathrm{W}$ (mainly impacting the self-heating impedances) and the center-to-center pitch $\mathrm{L}_{\mathrm{GG}}$ between adjacent gates (influencing the mutual counterparts), both represented in Fig. 3. The estimation grid needed for the proposed approach comprises the following combinations of $\left(\mathrm{W}, \mathrm{L}_{\mathrm{GG}}\right)=(75,15),(75,30),(75,45),(112.5,15),(112.5$, $30),(112.5,45),(150,15),(150,30),(150,45) \mu \mathrm{m}$ (estimation points). The validation points, which are used to test the model accuracy over design space points not used for its generation, are $\left(\mathrm{W}, \mathrm{L}_{\mathrm{GG}}\right)=(93.75,22.5),(93.75,37.5),(131.25,22.5)$, (131.25, 37.5) $\mu \mathrm{m}$.

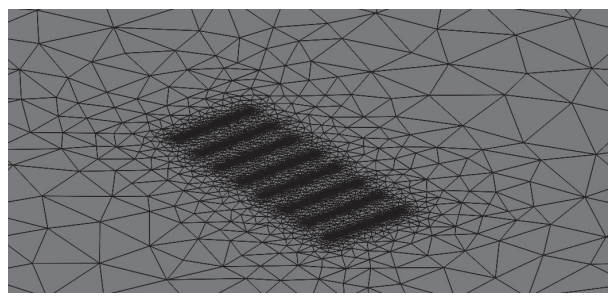

Fig. 2. Comsol mesh for a multi-gate HEMT under test.

Once the macromodel has been extracted, the CPU time needed to perform a time domain simulation of the thermal impedance matrix for assigned geometrical parameters is only $0.22 \mathrm{~s}$ on a normal PC equipped with an Intel Core2 Extreme CPU Q9300 2.53GHz and 8GB RAM, with a significant gain

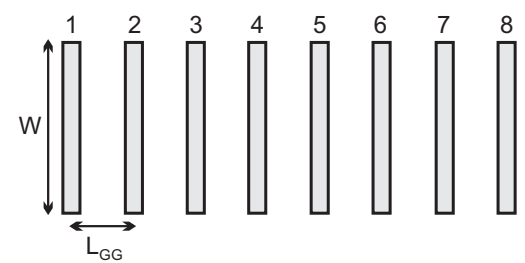

Fig. 3. Schematic top-view representation of the HEMT layout illustrating the gate fingers, the gate width $\mathrm{W}$ and spacing $\mathrm{L}_{\mathrm{GG}}$.

compared to the time/memory required by a conventional approach since a parameterized macromodel prevents generating a new mesh and evaluating a new impedance matrix by numerical simulations for a layout variation. In order to quantify the accuracy of the proposed approach in both the identification and verification stages, the following error can be suitably defined:

$$
\begin{array}{r}
\operatorname{Err}_{r e l}\left(\mathrm{Z}_{\mathrm{ij}}\right)=\max _{t}\left[100 \cdot\left|\frac{\mathrm{Z}_{\mathrm{ij}}(\mathrm{t})-\mathrm{Z}_{\mathrm{ij}, \text { model }}(\mathrm{t})}{\mathrm{Z}_{\mathrm{ij}}(\mathrm{t})}\right|\right] \cdot \frac{\mathrm{R}_{\mathrm{ij}}}{\mathrm{R}_{\max }} \\
\text { for } \mathrm{t} \geq \mathrm{t}^{*}, \mathrm{Z}_{\mathrm{ij}}\left(\mathrm{t}^{*}\right)=0.3 \mathrm{R}_{\mathrm{ij}}, \mathrm{R}_{\max }=\max _{i j}\left(\mathrm{R}_{\mathrm{ij}}\right)
\end{array}
$$

Fig. 4 depicts the comparison between the FEM data and the macromodel output at the estimation points (a) for the self-heating term $\mathrm{Z}_{11}(\mathrm{t})$ at $\mathrm{L}_{\mathrm{GG}}=30 \mu \mathrm{m}$ and $\mathrm{W}=75,112.5$, $150 \mu \mathrm{m}$, and (b) for the mutual impedances $Z_{12}(\mathrm{t})$ and $Z_{13}(\mathrm{t})$ at $\mathrm{W}=112.5 \mu \mathrm{m}$ and $\mathrm{L}_{\mathrm{GG}}=15,30,45 \mu \mathrm{m}$. At the estimation points used for the model generation, the maximum relative error for the thermal impedance matrix as defined in (13) is found to be less than $1.1 \%$, while the steady-state error, i.e., error (13) for the last time sample, is in the order of $0.1 \%$.
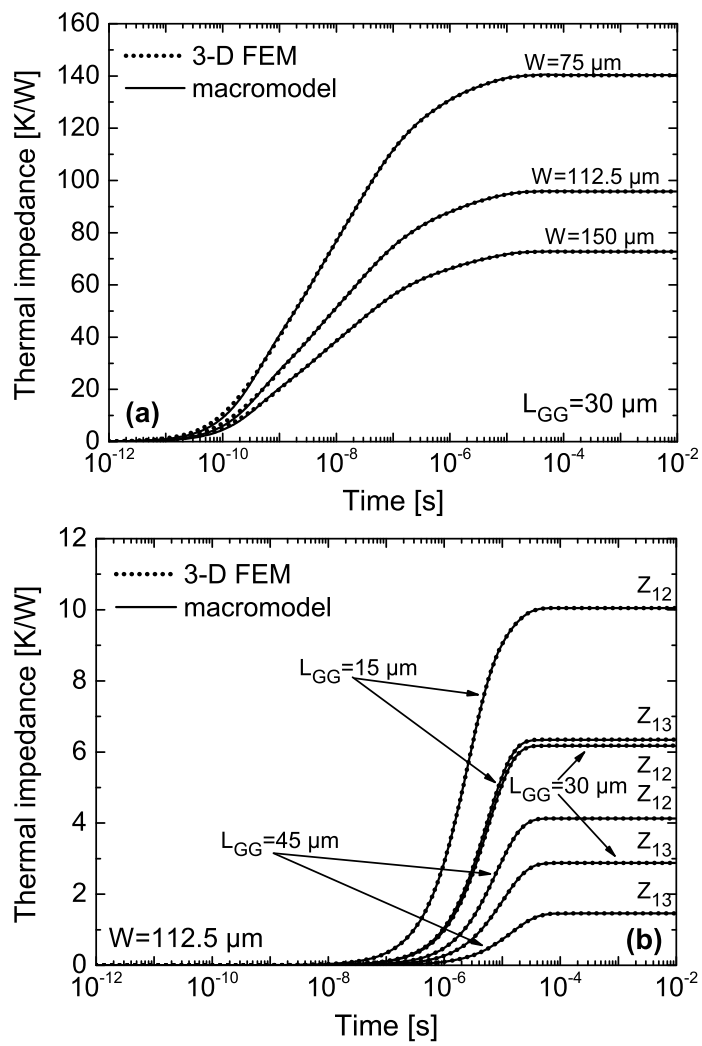

Fig. 4. Comparison between 3-D FEM (dotted lines) and macromodel results (solid) at the estimation points: (a) self-heating thermal impedance $\mathrm{Z}_{11}(\mathrm{t}$ ) for transistors with $\mathrm{L}_{\mathrm{GG}}=30 \mu \mathrm{m}$ and $\mathrm{W}=75,112.5,150 \mu \mathrm{m}$; (b) mutual thermal impedances $\mathrm{Z}_{12}(\mathrm{t})$ and $\mathrm{Z}_{13}(\mathrm{t})$ for transistors with $\mathrm{W}=112.5 \mu \mathrm{m}$ and $\mathrm{L}_{\mathrm{GG}}=15$, $30,45 \mu \mathrm{m}$. 
Fig. 5 shows a similar comparison for the validation points, depicting (a) $\mathrm{Z}_{11}$ (t) at $\mathrm{L}_{\mathrm{GG}}=22.5 \mu \mathrm{m}$ and $\mathrm{W}=93.75,131.25 \mu \mathrm{m}$, and (b) $\mathrm{Z}_{12}(\mathrm{t})$ and $\mathrm{Z}_{13}(\mathrm{t})$ at $\mathrm{W}=131.25 \mu \mathrm{m}$ and $\mathrm{L}_{\mathrm{GG}}=22.5$, $37.5 \mu \mathrm{m}$. In spite of the coarseness of the estimation grid (composed by 9 points only), a good agreement is obtained at the validation points as well: the maximum relative error (13) for all the elements of the thermal impedance matrix was found to be lower than $2.2 \%$.
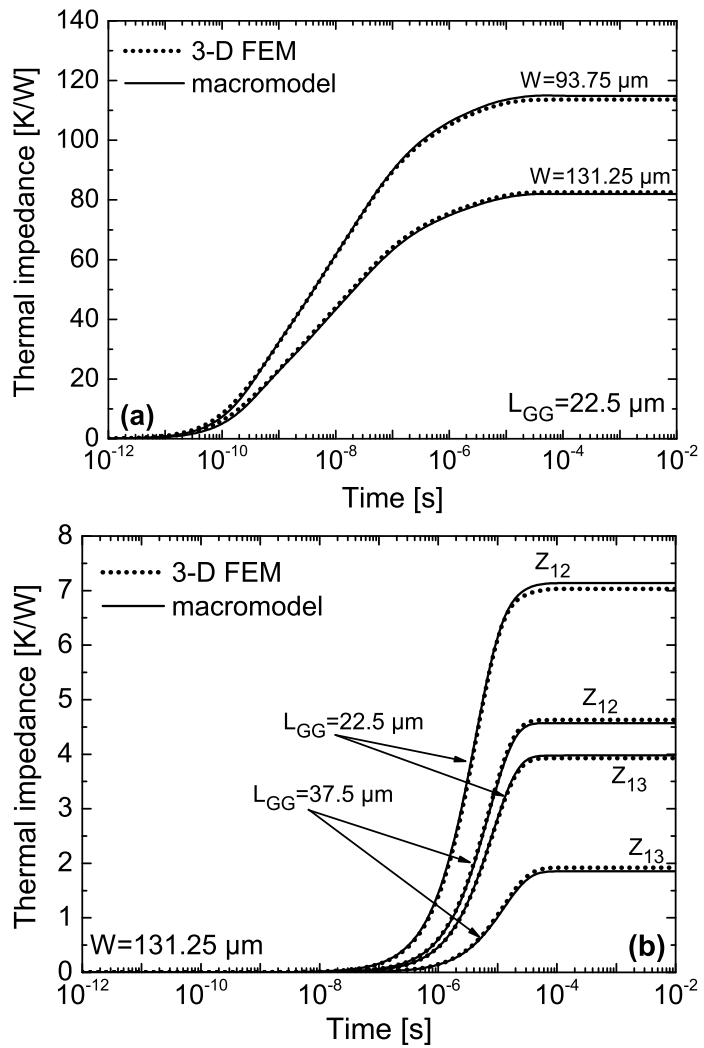

Fig. 5. Comparison between 3-D FEM (dotted lines) and macromodel results (solid) at the validation points: (a) self-heating thermal impedance $\mathrm{Z}_{11}(\mathrm{t}$ ) for transistors with $\mathrm{L}_{\mathrm{GG}}=22.5 \mu \mathrm{m}$ and $\mathrm{W}=93.75,131.25 \mu \mathrm{m}$; (b) mutual thermal impedances $\mathrm{Z}_{12}(\mathrm{t})$ and $\mathrm{Z}_{13}(\mathrm{t})$ for transistors with $\mathrm{W}=131.25 \mu \mathrm{m}$ and $\mathrm{L}_{\mathrm{GG}}=22.5,37.5 \mu \mathrm{m}$.

\section{CONCLUSIONS}

In this paper, a parameterized macromodeling strategy has been exploited for a fast prediction of the influence of design features on the dynamic thermal behavior of electronic components and systems with multiple heat sources. A pre-processing step on the frequency-domain data samples of the thermal impedance matrix is used to improve the macromodel accuracy and limit the number of numerical thermal simulations needed to build the macromodel. An 8-finger AlGaN/GaN HEMT suffering from radical thermal effects has been considered as a case-study. The estimation grid data samples needed to build the parameterized macromodel have been computed by numerically determining the thermal impedance matrix for 9 combinations of gate width and gate-to-gate pitch using 3-D FEM simulations. It has been found that the macromodel allows reaching a good accuracy in all 4 validation points; in particular, an error lower than $2.2 \%$ has been obtained in assessing all the thermal impedances. Moreover, the evaluation of the whole impedance matrix requires much less than 1 $\mathrm{s}$, whereas several hours would be needed by performing conventional numerical simulations. This study witnesses that the proposed methodology allows drastically reducing the number of computationally onerous 3-D simulations required to analyze the thermal behavior of electronic components and systems in an assigned technology. As a result, various design tasks can be quickly tackled and solved.

\section{ACKNOWLEDGMENT}

This work was partially supported by the Research Foundation Flanders (FWO-Vlaanderen). Francesco Ferranti is a Post-Doctoral Research Fellow of FWO-Vlaanderen.

\section{REFERENCES}

[1] M. Pedram and S. Nazarian, "Thermal modeling, analysis, and management in VLSI circuits: Principles and methods," Proc. IEEE, vol. 94, no. 8, pp. 1487-1501, 2006.

[2] Y. Zhan, S. V. Kumar, and S. S. Sapatnekar, "Thermally-aware design," Found. Trends Electron. Design Autom., vol. 2, no. 3, pp. 255-370, 2007.

[3] V. d'Alessandro, M. de Magistris, A. Magnani, N. Rinaldi, and S. Russo, "Dynamic electrothermal macromodeling: an application to signal integrity analysis in highly integrated electronic systems," IEEE Trans. Compon. Packag. Manuf. Technol., vol. 3, no. 7, pp. 1237-1243, 2013.

[4] F. Ferranti, L. Knockaert, and T. Dhaene, "Guaranteed passive parameterized admittance-based macromodeling," IEEE Trans. Adv. Packag., vol. 33, no. 3, pp. $623-629,2010$.

[5] — , "Parameterized S-parameter based macromodeling with guaranteed passivity," IEEE Microw. Wireless Compon. Lett., vol. 19, no. 10, pp. 608-610, 2009.

[6] P. Triverio, M. Nakhla, and S. Grivet-Talocia, "Passive parametric macromodeling from sampled frequency data," in Proc. IEEE 14th Workshop on Signal Propagation on Interconnects (SPI), 2010, pp. 117 -120 .

[7] — "Passive parametric modeling of interconnects and packaging components from sampled impedance, admittance or scattering data," in Proc. Electronic System-Integration Technology Conference (ESTC), 2010, pp. 1-6.

[8] F. Ferranti, L. Knockaert, and T. Dhaene, "Passivity-preserving parametric macromodeling by means of scaled and shifted state-space systems," IEEE Trans. Microw. Theory Tech., vol. 59, no. 10, pp. 2394-2403, 2011.

[9] F. Ferranti, T. Dhaene, and L. Knockaert, "Compact and passive parametric macromodeling using reference macromodels and positive interpolation operators," IEEE Trans. Compon. Packag. Manuf. Technol., vol. 2, no. 12, pp. 2080 -2088, 2012.

[10] R. A. Horn and C. R. Johnson, Topics in Matrix Analysis. Cambridge University Press, 1994.

[11] L. F. Eastman and U. K. Mishra, "The toughest transistor yet," IEEE Spectrum, vol. 39, no. 5, pp. 28-33, 2002.

[12] M. Peroni, P. Romanini, A. Pantellini, L. Mariucci, A. Minotti, G. Ghione et al., "Design, fabrication, and characterization of $\Gamma$ gate GaN HEMT for high-frequency/wide-band applications," in Proc. WOCSDICE, 2007, pp. 371-378.

[13] S. Russo, V. d'Alessandro, M. Costagliola, G. Sasso, and N. Rinaldi, "Analysis of the thermal behavior of AlGaN/GaN HEMTs," Materials Science and Engineering: B - Advanced Functional Solid-State Materials, vol. 177, no. 15, pp. 1343-1351, 2012.

[14] Comsol Multiphysics 3.5a, User's Manual, COMSOL AB, Stockholm, Sweden, 2008 